\title{
Optical-NIR spectroscopy of the puzzling $\gamma$-ray source 3FGL 1603.9-4903/PMN J1603-4904 with X-Shooter ${ }^{\star}$
}

\author{
P. Goldoni ${ }^{1}$, S. Pita ${ }^{1}$, C. Boisson ${ }^{2}$, C. Müller ${ }^{3,4}$, T. Dauser ${ }^{5}$, I. Jung ${ }^{6}$, F. Krauß ${ }^{4,5}$, J.-P. Lenain ${ }^{7}$, and H. Sol ${ }^{2}$ \\ 1 APC, Univ. Paris Diderot, CNRS/IN2P3, CEA/Irfu, Obs. de Paris, Sorbonne Paris Cité, 75013 Paris, France \\ e-mail: goldoni@apc .in2p3.fr \\ 2 LUTH, Obs. de Paris, CNRS, Université Paris Diderot, PSL, 75006 Paris, France \\ 3 Department of Astrophysics/IMAPP, Radboud University Nijmegen, PO Box 9010, 6500 GL Nijmegen, The Netherlands \\ 4 Institut für Theoretische Physik und Astrophysik, Universität Würzburg, Am Hubland, 97074 Würzburg, Germany \\ 5 Dr. Remeis Sternwarte \& ECAP, Universität Erlangen-Nürnberg, Sternwartstrasse 7, 96049 Bamberg, Germany \\ ${ }^{6}$ ECAP, Universität Erlangen-Nürnberg, Erwin-Rommel-Str. 1, 91508 Erlangen, Germany \\ 7 Sorbonne Universités, UPMC Univ. Paris 06, Univ. Paris Diderot, Sorbonne Paris Cité, CNRS, \\ Laboratoire de Physique Nucléaire et de Haute Energies (LPNHE), 4 Place Jussieu, 75252 Paris Cedex 5, France
}

Received 16 October 2015 / Accepted 17 December 2015

\section{ABSTRACT}

\begin{abstract}
Context. The Fermi/LAT instrument has detected about two thousand extragalactic high energy $(E \geq 100 \mathrm{MeV}) \gamma$-ray sources. One of the brightest is 3FGL J1603.9-4903; it is associated to the radio source PMN J1603-4904. Its nature is not yet clear, it could be either a very peculiar BL Lac or a compact symmetric object radio source which are considered as the early stage of a radio galaxy. The latter, if confirmed, would be the first detection in $\gamma$-rays for this class of objects. A redshift $z=0.18 \pm 0.01$ has recently been claimed on the basis of the detection of a single X-ray line at $5.44 \pm 0.05 \mathrm{keV}$ which has been interpreted as a $6.4 \mathrm{keV}$ (rest frame) fluorescent line.

Aims. We aim to investigate the nature of 3FGL J1603.9-4903/PMN J1603-4904 using optical-to near-IR (NIR) spectroscopy.

Methods. We observed PMN J1603-4904 with the UV-NIR VLT/X-Shooter spectrograph for two hours. We extracted spectra in the visible and NIR range that we calibrated in flux and corrected for telluric absorption. We systematically searched for absorption and emission features.

Results. The source was detected starting from $\sim 6300 \AA$ down to $24000 \AA$ with an intensity similar to that of its 2 MASS counterpart and a mostly featureless spectrum. The continuum lacks absorption features and thus is non-stellar in origin and most likely non-thermal. In addition to this spectrum, we detected three emission lines that we interpret as the H $\alpha$-[NII] complex, the [SII] $\lambda, \lambda 6716,6731$ doublet and the [SIII] $\lambda 9530$ line; we obtain a redshift estimate of $z=0.2321 \pm 0.0004$. The line ratios suggest that a LINER/Seyfert nucleus powers the emission. This new redshift measurement implies that the X-ray line previously detected should be interpreted as a $6.7 \mathrm{keV}$ line which is very peculiar.
\end{abstract}

Key words. galaxies: active - galaxies: individual: PMNJ1603-4904 - gamma rays: galaxies - galaxies: distances and redshifts

\section{Introduction}

The Large Area Telescope (LAT) onboard the Fermi $\gamma$-ray space telescope has dramatically improved our knowledge of the extragalactic $\gamma$-ray sky at high energies $(\mathrm{HE} ; E>100 \mathrm{MeV})$; it has expanded the number of known $\gamma$-ray sources by an order of magnitude (Acero et al. 2015). In particular, Fermi/LAT provides important insights into the understanding of mechanisms at play in blazars which represent the majority $(\sim 85 \%)$ of the 2000 identified or associated sources of the 3FGL catalog (Acero et al. 2015). The fact that several of the detected blazars are bright $\gamma$-ray sources has allowed determining spectral properties and studying temporal variability at different timescales with an accuracy that is unprecedented for this energy domain. Moreover, the brightest and hardest sources are also detected by the latest generation of very high energy (VHE; $E>100 \mathrm{GeV}$ ) ground based instruments such as

$\star$ Based on observations collected at the European Organisation for Astronomical Research in the Southern Hemisphere, Chile, under program 095.B-0400(A). The raw FITS data files are available in the ESO archive.
H.E.S.S., MAGIC, or VERITAS (Aharonian et al. 2008), providing stronger constraints on the energetics and the radiation fields of objects. These VHE sources will become much more numerous in the next decade with the deployment of the CTA array (Actis et al. 2011).

Fermi/LAT also detected a handful of other extragalactic sources, among them 14 radio galaxies (Ackermann et al. 2015). These sources are thus a minority in the HE sky, but they can provide general insights into the jet structure in AGNs. Their faintness in the $\gamma$-ray domain prevents a precise spectral and temporal characterization. Therefore, the detection of bright $\gamma$-ray extragalactic sources that do not belong to the blazar class could allow a significant improvement in our knowledge of the particle acceleration and radiation mechanisms responsible for $\gamma$-ray emission.

One of the brightest sources detected by Fermi/LAT in the HE $\gamma$-ray sky is 3FGL J1603.9-4903. Its spectrum at the highest energies ( $E>10 \mathrm{GeV}$; Ackermann et al. 2013) shows a photon index of $1.96 \pm 0.14$, which makes this source a very probable high signal-to-noise detection in the VHE regime. The only radio counterpart which can be found well within the $95 \%$ Fermi/LAT 
error box is PMN J1603-4904. On the basis of its $\gamma$-ray properties the source has been classified as a low-synchrotron peaked (LSP) BL Lac (Ackermann et al. 2015). Notably, the analysis of the first two years of Fermi LAT observations indicated that the source had a very low variability (Nolan et al. 2012) while the results of the first four years suggest a high variability (Ackermann et al. 2015). The source lies at $\sim 2.5^{\circ}$ degrees from the Galactic plane in a heavily absorbed region, it has a weak optical counterpart (Müller et al. 2014) and a moderately bright NIR counterpart, 2MASSJ16035069-4904054. The only spectroscopic observation of the source was performed by Shaw et al. (2013) between $\sim 4000$ and $\sim 7000 \AA$ with NTT/EFOSC2 and only $20 \mathrm{~min}$ of exposure. No emission or absorption lines were detected; this result is consistent with the LSP classification.

Recently, Müller et al. (2014) showed that the source NIR and MIR emission is dominated by thermal radiation that is not consistent with stellar emission and many times brighter than expected for a typical blazar spectral energy distribution (SED) in this range. They also found that the emission in the radio band is extended on $\sim 0.01^{\prime \prime}$ double-lobed and symmetric. The symmetry of the radio lobes is difficult to explain if the jet is directed toward the observer as in blazars. Their observations suggest two possible scenarios for the source counterpart. One is a very peculiar blazar (most probably a BL Lac) lacking some of the standard properties of the class such as fast variability. The second is a radio galaxy whose jet lies in a plane roughly perpendicular to the line of sight. Because the small spatial extension of the radio emission is not compatible with a radio galaxy, they suggested that PMN J1603-4904 might be a compact symmetric object (CSO) radio source. This class of sources is defined by the limited spatial extension of its members and is considered to be the early stage of a radio galaxy (O'Dea 1998). While this scenario explains the radio phenomenology well, no CSO has been firmly detected in $\gamma$-rays as yet, although models have been suggested (Stawarz et al. 2008).

Finally in deep XMM and Suzaku X-ray observations of PMN J1603-4904 (Müller et al. 2015), the source was clearly detected and modeled with an absorbed power-law spectrum with a spectral index $\Gamma=2.07_{-0.1}^{+0.4}$ and a Gaussian line centered at $5.44 \pm 0.05 \mathrm{keV}$. Interpreting the line as a $6.4 \mathrm{keV}$ fluorescence line, which is the most prominent line in the $\mathrm{X}$ rays because it yields the highest fluorescence, they estimated the redshift at $0.18 \pm 0.01$. It is remarkable that, in addition to the Galactic absorption in the direction of the source, $(6.3 \times$ $10^{21} \mathrm{~cm}^{-2}$; Kalberla et al. 2005), an intrinsic absorption three times as strong, $N_{\mathrm{H}}=2.05_{-0.12}^{+0.14} \times 10^{22} \mathrm{~cm}^{-2}$ was detected.

The nature of PMN J1603-4904 is thus not completely determined and information on its properties in the optical/NIR domain is clearly lacking. We report on the results on optical/NIR spectroscopy with the X-Shooter spectrograph aimed to gather more information on its nature.

For all calculations, we used a cosmology with $\Omega_{\mathrm{M}}=0.27$, $\Omega_{\Lambda}=0.73$ and $H_{0}=71 \mathrm{~km} \mathrm{~s}^{-1} \mathrm{Mpc}^{-1}$. All wavelengths are measured in vacuum.

\section{Observations and results}

The X-Shooter spectrograph (Vernet et al. 2011) is a singleobject medium-resolution échelle spectrograph whose main characteristic is an unprecedented simultaneous wavelength coverage from $3000 \AA$ to $24000 \AA$. This is obtained by splitting the light using dichroics into three arms: UVB $(\lambda=3000-5600 \AA)$, VIS $(\lambda=5500-10200 \AA)$, and NIR $(\lambda=10000-24000 \AA)$. Its resolution $\mathcal{R}$ is between 3000 and 17000 depending on arm and slit width. For these observations, we chose slit widths of 1.3", $1.3^{\prime \prime}$ and $1.2^{\prime \prime}$ for the UVB, VIS, and NIR arms, which resulted in $\mathcal{R} \sim 4000,6700$, and 3900 respectively.

Two observations were performed, the first on 2015 April 30 and the second about two weeks later on 2015 May 13. The conditions were photometric in the first night and clear in the second. The average airmass and seeing at zenith of the two nights were 1.27 and 1.14 and $0.76^{\prime \prime}$ and $0.57^{\prime \prime}$ respectively. The exposures were taken using the nodding-along-the-slit technique in a standard ABBA sequence with an offset of 5" between exposures of about $690 \mathrm{~s}$ each (the exposure time was slightly different for each arm). Each observation was preceded or followed by an observation of a telluric standard star at similar airmass. The faint optical emission presents an elliptical shape extended on $\sim 2.5^{\prime \prime}$ in the east-west direction and $\sim 1.4^{\prime \prime}$ in the north-south direction (Müller et al. 2014). Due to nearby sources, the slit was oriented conservatively toward the north, thereby covering only the central 1.2" (i.e. about half) of the source.

We processed the spectra using version 2.0 of the X-Shooter data reduction pipeline (Goldoni et al. 2006). A description of the detailed reduction and flux calibration process can be found in Pita et al. (2014). The spectra were extracted with a binning of $0.2 \AA$ in UVB and VIS data and $0.5 \AA$ in the NIR data. They were then telluric corrected and flux calibrated using the SpeXtool IDL procedure (Vacca et al. 2003) and the SIMBAD magnitudes of the telluric standard stars to set the absolute flux scale. No variability was detected between the two observations, therefore the two spectra were averaged.

In the NIR data a bright source with magnitudes compatible with those of the 2MASS counterpart was detected. The same source was also detected in the VIS arm down to $\sim 6300 \AA$. Integrating the VIS spectrum (Pita et al. 2014), we obtained an equivalent magnitude $r^{\prime}=22.4 \pm 0.2$. As a result of the slit position (see above), this value is only applicable to the central half of the source. In previous observations (Müller et al. 2014) the optical counterpart appeared to have roughly a constant surface brightness and $r^{\prime} \sim 24$ for the eastern part (i.e. one third of the source). While the comparison is difficult because the measurements were taken in different parts of the source, this great discrepancy (of a factor $\sim 3$ taking into account the size difference) implies that the region of the source that was observed has varied in the $r$ ' band between the two observations. The object was not detected in the UVB data.

We corrected for Galactic absorption using the maps of Schlafly \& Finkbeiner (2011) and obtained $E(B-V)=2.19$ for the direction of PMN J1603-4904. This translates into $A_{V}=6.8$ using the usual relationship $R_{V}=A_{V} / E(B-V)=3.1$ (Rieke $\&$ Lebofsky 1985). We then dereddened the spectra using the parametrization of Fitzpatrick (1999), the result is shown in Fig. 1.

The detected spectrum is mostly smooth, it does not show narrow lines or the strong absorption lines typical of an elliptical galaxy. The unabsorbed spectrum is clearly of non-stellar origin; it can be fit with two power laws $\left(F \propto \lambda^{\alpha}\right)$ with a break at $\sim 12000 \AA$. The energy indices are $\sim-0.4 \pm 0.1$ for $\lambda \leq 12000 \AA$ and $-1.1 \pm 0.1$ for $\lambda \geq 12000 \AA$ (see Fig. 1). Alternatively the NIR part of the spectrum may also be fit with a blackbody at $T \sim 1600 \mathrm{~K}$ as in Müller et al. (2014) but the fit is noticeably worse. From this analysis, it appears that the continuum is nonthermal, at least in the VIS part of the spectrum.

We did not find any spectral feature consistent with the redshift found by Müller et al. (2015). However, we found 


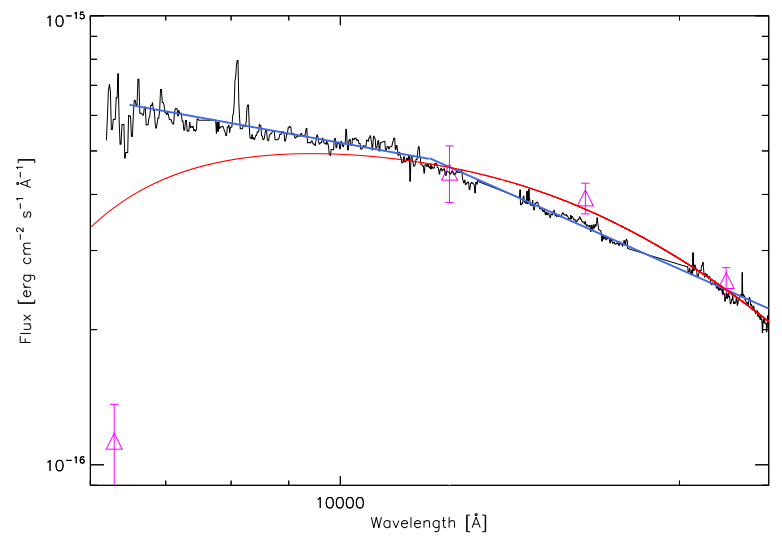

Fig. 1. X-Shooter dereddened spectrum of PMNJ1603-4904, along with 2MASS photometry and optical photometry (Müller et al. 2014; magenta, triangles with error bars). The data were dereddened using the parametrization of Fitzpatrick (1999) and the $E(B-V)$ of Schlafly \& Finkbeiner (2011). We superimposed on the spectra a continuum made by two power laws of index -0.4 and -1.1 in blue and a blackbody at $T=1600 \mathrm{~K}$ (red). The blackbody cannot reproduce the spectrum at wavelengths shorter than $\sim 12000 \AA$.

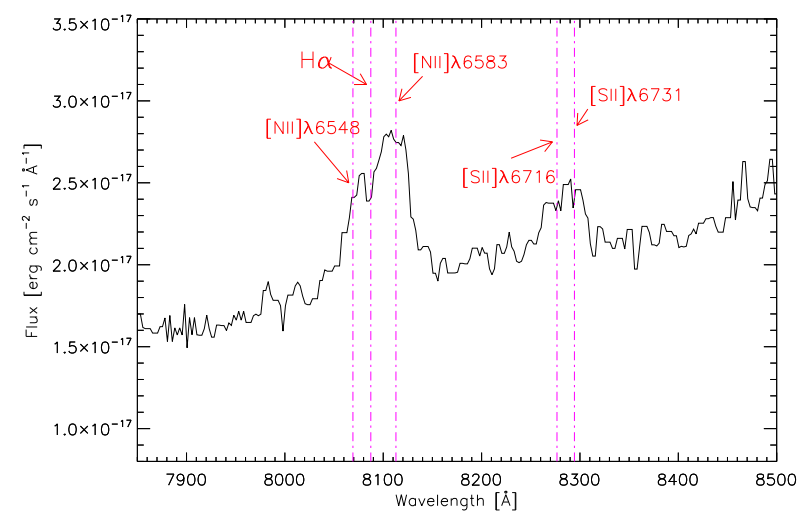

Fig. 2. Section of the flux-calibrated and telluric-corrected X-Shooter spectrum binned to $2 \AA$ bins displaying the $\mathrm{H} \alpha$-[NII] complex and the [SII] $\lambda \lambda$ 6716, 6731 doublet. The vertical magenta (dot-dashed) lines show the positions of the feature components (see text).

one bright, asymmetric, emission feature in the VIS spectrum, centered around $8100 \AA$ and a fainter feature centered around $8250 \AA$ (Fig. 2). In the NIR spectrum we found a much fainter emission line centered around $11700 \AA$.

These three features can be interpreted as the $\mathrm{H} \alpha$-[NII] complex (formed by the combination of $\mathrm{H} \alpha$, [NII] $\lambda 6583$ and [NII] $\lambda$ 6548), the doublet [SII] $\lambda \lambda$ 6716, 6731 and [SIII] $\lambda 9530$ at a redshift $\sim 0.23$, respectively. The line [SIII] $\lambda$ 9069, associated with [SIII] $\lambda 9530$ and usually weaker by a factor 2.44 , is not detected, this is consistent with its weakness and its position in a wavelength region that is heavily contaminated by telluric absorption. At this redshift the optical luminosity is $1.0 \times 10^{45}$ ergs/s and, taking into account the results of the 3FGL catalog, the $\gamma$-ray luminosity is $8.5 \times 10^{45} \mathrm{ergs} / \mathrm{s}$. After rebinning the spectra at $2 \AA$, we measured the rest-frame equivalent width by integrating the flux of the three features taking into account errors in the continuum placement as in Sembach \& Savage (1992) and obtaining $28.6 \pm 3.3 \AA, 10.2 \pm 1.9 \AA$ and $5.7 \pm 1.0 \AA$. The first two values are much higher than the usually quoted limit of $5 \AA$ for the maximum EW of an emission line in a BL Lac object (Urry \& Padovani 1995).
Fitting a single Gaussian to the $11700 \AA$ feature, we estimate a precise redshift of $z=0.2321 \pm 0.0004$. The fit of the [SIII] $\lambda 9530$ results in $F W H M \sim 1300 \mathrm{~km} \mathrm{~s}^{-1}$, but the weakness of the line and the noisy background render this determination uncertain. To estimate the parameters of the remaining lines we constrained the velocity widths of the detected doublets [NII] $\lambda \lambda 6583,6548$ and of [SII] $\lambda \lambda 6716,6731$ to be the same and the flux ratio [NII] $\lambda$ 6583/[NII] $\lambda 6548$ equal to 3 . Formally acceptable but weakly constrained fits can be obtained with this configuration; the widths of the lines are between 1000 and $1800 \mathrm{~km} \mathrm{~s}^{-1}$. With this decomposition we also obtain $E W(\mathrm{H} \alpha)=9.1 \pm 2.0 \AA$ and $E W([\mathrm{NII}])=16.8 \pm 3.6 \AA$.

Finally we reanalyzed the X-ray spectra presented in Müller et al. (2015) using the new redshift, the results are essentially unchanged with spectral index $\sim 2.0$ and intrinsic $N_{\mathrm{H}} \sim 2.3 \times 10^{22}$ $\mathrm{cm}^{-2}$. In this fit the $\mathrm{X}$-ray line is centered at $6.7 \mathrm{keV}$, which is very peculiar, but not impossible for this kind of source. We also note that a worse fit can be obtained with Galactic absorption $N_{H}=2 \times 10^{22} \mathrm{~cm}^{-2}$, but these column densities are quite rare in this region of the sky, because only $7 \%$ of the sightlines in a cone of 10 degrees radius around PMN J1603-4904 have this absorption in the LAB survey (Kalberla et al. 2005). We therefore consider this possibility unlikely.

\section{Discussion}

We have detected the optical and NIR emission of the central region of the optical counterpart of PMN J1603-4904with $\mathrm{X}$-Shooter observations. The continuum spectrum is characterized by non-stellar, most likely nonthermal, emission that displays a spectral break around $12000 \AA$. In addition of the continuum emission we detected three emission lines consistent with the $\mathrm{H} \alpha$-[NII] complex, the doublet [SII] $\lambda \lambda$ 6716, 6731 and $[\mathrm{SIII}] \lambda 9530$ at redshift $0.2321 \pm 0.0004$. The equivalent width of the lines is much higher than $5 \AA$, the commonly used threshold between BL Lacs and FSRQs. Blazars can sometimes cross this limit, BL Lac itself has displayed EWs up to $15 \AA$ (Vermeulen et al. 1995). Ruan et al. (2014) investigated this phenomenon, and found only six cases of similar transitions in 602 unique repeat pairs of SDSS spectra of 354 blazars. This transition is therefore improbable, but not impossible. In a more physical classification scheme Ghisellini et al. (2011) separated BL Lacs from FSRQs using the Fermi 2FGL $\gamma$-ray luminosity and spectral index. In this scheme, PMN J1603-4904 with $8 \times 10^{45} \mathrm{erg} / \mathrm{s}$ and $1.12 \pm 0.025$ would marginally be classified as a BL Lac. (see their Fig. 1). However the transition between the two classes is not sharp because sources classified as BL Lac and FSRQs can be found on both sides of the limit. We conclude that PMN J1603-4904 can be tentatively classified as a blazar, but more observations are needed to determine whether it is a BL Lac or an FSRQ.

We fitted the lines using Gaussian functions for each atomic species. Using this decomposition, we obtained a ratio of $[\mathrm{NII}] / \mathrm{H} \alpha=1.3 \pm 0.25$. This value is much greater than what can be produced in star-forming regions, for instance $\sim 0.4$ (see, e.g. Baldwin et al. 1981) and can be produced by AGN radiation (Kewley et al. 2013). We then computed the ratio ([SII] $\lambda$ $6716+[\mathrm{SII}] \lambda 6731) / \mathrm{H} \alpha=0.59 \pm 0.27$ and the ratio ([SIII] $\lambda$ $9069+[\mathrm{SIII}] \lambda$ 9530) $/ \mathrm{H} \alpha=0.47 \pm 0.23$. The latter was computed assuming that $[\mathrm{SIII}] \lambda 9530 /[\mathrm{SIII}] \lambda 9069=2.44$. According to Diaz et al. (1985, see their Fig. 5), these ratios are produced when the ionization is due to the radiation of an AGN.

The fitted FWHMs lie between $\sim 1000 \mathrm{~km} \mathrm{~s}^{-1}$ and $1800 \mathrm{~km} \mathrm{~s}^{-1}$ and are much greater than the typical FWHMs 
(200-300 $\mathrm{km} \mathrm{s}^{-1}$; Veron et al. 1997) of emission lines in starforming regions. These line widths are intermediate between the typical widths of lines originating in broad line regions and narrow line regions (Peterson 2006). These widths can nonetheless be produced by the combination of several independent narrow components as occurs in the nearby Seyfert 2 galaxy NGC 1068 (Axon et al. 1998). We also searched for evidence of an additional broad ( $F W H M \geq 2000 \mathrm{~km} \mathrm{~s}^{-1}$ ) $\mathrm{H} \alpha$ component and we found that adding this component does not significantly improve the fit. We conclude that the lines are most likely powered by a LINER or Seyfert nucleus.

To estimate a limit to the SFR from the absence of narrow lines, we added a narrow $\left(500 \mathrm{~km} \mathrm{~s}^{-1}\right) \mathrm{H} \alpha$ line to our fit that is representative of the emission from HII regions. Our fit does not reproduce the $\mathrm{H} \alpha$-[NII] complex anymore if the narrow-line $\mathrm{H} \alpha$ luminosity becomes greater than $7 \times 10^{42} \mathrm{erg} / \mathrm{s}(2 \sigma$ upper limit $)$ implying $S F R<3.8 M_{\odot} / y r$ (Kennicutt 1998). Therefore the central region of PMN J1603-4904 does not experience a major starburst. The detection of probably variable non-stellar emission together with moderately bright emission lines suggests that the object is a radio galaxy powered by a LINER/Seyfert nucleus. The rather high intrinsic X-ray absorption column density $(2 \times$ $10^{22} \mathrm{~cm}^{-2}$ ) is typical of a Seyfert 1.8-1.9 nucleus (Risaliti et al. 1999) and fits this scenario well.

In this case the high-energy (optical to $\gamma$-rays) radiation would be produced through inverse Compton upscattering of the photon fields by the particles in the jet (Stawarz et al. 2008). The very small extension in radio $\left(\sim 0.01^{\prime \prime}\right.$ are $\sim 40 \mathrm{pc}$ at $\left.z=0.2321\right)$, typical of CSOs, may be explained if PMN J1603-4904 is a very young radio source whose jet has not had the time to expand. If this were correct, this would be the first detection of a $\gamma$-ray emitting CSO. Alternatively, PMN J1603-4904 could be interpreted as a blazar with a peculiarly high X-ray absorption and significant iron line emission. In this scenario, the additional X-ray absorption at the galaxy redshift could be due to a dense interstellar cloud in the path of the jet. A distant foreground cloud causes a similar column density in the peculiar BL Lac PKS 1413+135 (Perlman et al. 2002). However in the case of PMN J1603-4904 the presence of the $6.7 \mathrm{keV}$ line suggests that the cloud may be local.

In both scenarios the presence of a single $6.7 \mathrm{keV}$ line without an associated $6.4 \mathrm{keV}$ line is puzzling, however. One possible interpretation of this line would be that it is a reflection on a highly ionized thin cloud in the optical path of the X-ray emission. The cloud would be ionized by interaction of the jet with the ambient medium through collisional processes. In this case, the collisionally ionized plasma might even be able to produce the observed $6.7 \mathrm{keV}$ emission line without the need of an additional reflection component. Strong photoionization may also produce such a line, but a harder and brighter X-ray spectrum would be needed. A more detailed analysis of the origin of this line is beyond the scope of this paper.

\section{Conclusions}

PMN J1603-4904 has been suggested to be a very peculiar BL Lac or a young radio galaxy seen edge-on. We observed the central region of the optical counterpart with X-Shooter. Our main results are:

- We detected a non-stellar, most likely nonthermal, continuum that varied in the $r$ ' band with respect to earlier observations. In addition we detected three lines at a redshift consistent with $0.2321 \pm 0.0004$.
- The EW of the brightest line (the $\mathrm{H} \alpha$-[NII] complex) is much greater than $5 \AA$, but the properties of the source did not allow us to classify it as a BL Lac or as an FSRQ. The line ratios are consistent with the gas being excited by an AGN nucleus.

- We measured an upper limit to the star formation rate of 3.8 $M_{\odot} / \mathrm{yr}$ implying that no strong star formation occurs in the observed region.

These results, together with previous multiwavelength observations, led us to consider two possible scenarios for the nature of the source. In the first scenario, PMN J1603-4904 might be a radio galaxy of the CSO type. This would be the first object of its kind detected in $\gamma$-rays which would permit a great advancement in our understanding of the mechanism producing $\gamma$-ray radiation. In the second scenario, the source might be a blazar, whose X-ray emission is obscured through occultation by a dense intervening and possibly local cloud. More observations are needed to distinguish between these two scenarios. Radio and millimeter observations aimed at detecting $\mathrm{HI} 21 \mathrm{~cm}$ and molecular lines in absorption may also help determine the environment of this object. New, deeper optical spectroscopy of the totality of the source by changing the position angle of the slit would be particularly worthwhile. In the central region this would allow checking for variability of the continuum and lines. In the eastern and western parts, this would permit searching for the features of the host galaxy, which will give indications on its orientation with respect to the line of sight and to the radio source.

Acknowledgements. We thank the anonymous referee for a constructive report that helped improve the paper. We thank C. Martayan, M. Torres, T. Zafar, J. Pritchard and Th. Rivinius for performing the observations in service mode.

\section{References}

Acero, F., Ackermann, M., Ajello, M., et al. 2015, ApJS, 218, 23 Ackermann, M., Ajello, M., Allafort, A., et al. 2013, ApJS, 209, 34 Ackermann, M., Ajello, M., Atwood, W. B., et al. 2015, ApJ, 810, 14 Actis, M., Agnetta, G., Aharonian, F., et al. 2011, Exp. Astron., 32, 193 Aharonian, F., Buckley, J., Kifune, T., \& Sinnis, G. 2008, Rep. Prog. Phys., 71, 096901

Axon, D. J., Marconi, A., Capetti, A., et al. 1998, ApJ, 496, L75

Baldwin, J. A., Phillips, M. M., \& Terlevich, R. 1981, PASP, 93, 5

Diaz, A. I., Pagel, B. E. J., \& Wilson, I. R. G. 1985, MNRAS, 212, 737

Fitzpatrick, E. L. 1999, PASP, 111, 63

Ghisellini, G., Tavecchio, F., Foschini, L., \& Ghirlanda, G. 2011, MNRAS, 414, 2674

Goldoni, P., Royer, F., François, P., et al. 2006, in SPIE Conf. Ser., 6269

Kalberla, P. M. W., Burton, W. B., Hartmann, D., et al. 2005, A\&A, 440, 775

Kennicutt, Jr., R. C. 1998, ARA\&A, 36, 189

Kewley, L. J., Dopita, M. A., Leitherer, C., et al. 2013, ApJ, 774, 100

Müller, C., Kadler, M., Ojha, R., et al. 2014, A\&A, 562, A4

Müller, C., Krauß, F., Dauser, T., et al. 2015, A\&A, 574, A117

Nolan, P. L., Abdo, A. A., Ackermann, M., et al. 2012, ApJS, 199, 31

O'Dea, C. P. 1998, PASP, 110, 493

Perlman, E. S., Stocke, J. T., Carilli, C. L., et al. 2002, AJ, 124, 2401

Peterson, B. M. 2006, in Physics of Active Galactic Nuclei at all Scales, ed. D. Alloin, (Berlin: Springer Verlag), Lect. Notes Phys., 693, 77

Pita, S., Goldoni, P., Boisson, C., et al. 2014, A\&A, 565, A12

Rieke, G. H., \& Lebofsky, M. J. 1985, ApJ, 288, 618

Risaliti, G., Maiolino, R., \& Salvati, M. 1999, ApJ, 522, 157

Ruan, J. J., Anderson, S. F., Plotkin, R. M., et al. 2014, ApJ, 797, 19

Schlafly, E. F., \& Finkbeiner, D. P. 2011, ApJ, 737, 103

Sembach, K. R., \& Savage, B. D. 1992, ApJS, 83, 147

Shaw, M. S., Romani, R. W., Cotter, G., et al. 2013, ApJ, 764, 135

Stawarz, Ł., Ostorero, L., Begelman, M. C., et al. 2008, ApJ, 680, 911

Urry, C. M., \& Padovani, P. 1995, PASP, 107, 803

Vacca, W. D., Cushing, M. C., \& Rayner, J. T. 2003, PASP, 115, 389

Vermeulen, R. C., Ogle, P. M., Tran, H. D., et al. 1995, ApJ, 452, L5

Vernet, J., Dekker, H., D’Odorico, S., et al. 2011, A\&A, 536, A105

Veron, P., Goncalves, A. C., \& Veron-Cetty, M.-P. 1997, A\&A, 319, 52 